\title{
Technique to Enhance FDM 3D Metal Printing
}

\author{
Abhishek Kumar Singh and Sriram Chauhan
}

\begin{abstract}
D Printing is the new revolution of the manufacturing industries in $21^{\text {st }}$ century. The next move of innovation is to make it affordable enough to be hired for all domestic purpose also. This technology should reach every desk at a reasonable cost. The applications of $3 D$ printing is very wide, and most importantly in Metal Printing. In this research paper a different approach for 3D Metal Printing will be used. Moving from costly SLS to FDM (Fused Deposition Modelling) technology will make it affordable at every desk. The motif of this project is to make a 3D Metal Printer capable enough to produce tangible components out of metal whenever or wherever needed at affordable cost. This is done by introduction of Induction Heating to make the cartridges reusable. A brief introduction of SLS technology is given and then the article is focused on Technique to Enhance FDM 3D Metal Printing. Along with this, cost analysis of both FDM and SLS technology in 3D Metal Printing, and their advantages and disadvantages are discussed. We will find out the ways of gaining access to easy manufacturing of the printing device, enhanced speed of $3 D$ printing, extremely cheap cartridges (since the printer is a one-time investment but the cartridges aren't) and ability to recycle-reuse. Finally, we outline the enormous potential of FDM 3D Metal Printing.
\end{abstract}

Keywords--- 3D Metal Printing, Induction Heating, Fused Deposition Modelling, Desktop Printer, Cost Analysis, Affordable, Recyclable, Reusable Cartridge.

\section{INTRODUCTION}

3D printing is the process of additive manufacturing i.e. synthesis of a three dimensional object by means of addition of very thin layers of the prototype to be manufactured. Similarly, additive manufacturing of metal is called 3D Metal Printing. 3D Metal Printing is supposed to bring new revolution in this world. The motif of this project is to make a 3D Metal Printer capable enough to produce tangible components out of metal whenever and wherever needed at affordable cost [1]. By The end of this research paper we will be able to understand the conceptual design for production of a 3D metal printer capable enough to print $3 \mathrm{D}$ printed prototypes at the rates significantly cheaper than current technology.

When talking about 3D printing, many companies and researchers have focused on Speed, Mass Production, and Affordability. Apart from these factors, we will be enabling the methods to produce the job by recycling the scrap metals. Recyclability is the key advantage of this prototype. This capability is essentially required for the future where our prime

Abhishek Kumar Singh, Department of Mechanical Engineering, National Institute of Technology, Karaikal, India. E-mail: abhishek.kumar.singh.nitpy@gmail.com

Sriram Chauhan, Department of Mechanical Engineering, National Institute of Technology, India. E-mail:chauhan.sriram61@gmail.com DOI:10.9756/BIJIEMS.7574 focus will be on remodeling the prototype rather than building prototype with a new cartridge every time.

\section{CLASSIFICATION OF 3D PRINTING}

In the year 2010, as per the American Society for Testing and Materials (ASTM) group "ASTM F42-Additive Manufacturing", has categorized 3D printing in seven categories (Standard Terminology for Additive Manufacturing Technologies 2012) [11]. They are as follows:

- Directed Energy Deposition.

- $\quad$ Binder Jetting.

- Vat Photopolymerisation.

- Material Extrusion.

- Material Jetting.

- Sheet Lamination.

- Powder Bed Fusion.

Broadly they can be classified into three major types of 3D printing techniques based on the way they build up the layer for printing of prototype.

\section{A. SLA - Steriolithography}

It uses a photopolymer resin (molten plastic sensitive to UV light) in liquid tub which is struck by UV Laser focused at points of interest and solidifies that particular layer. This process is repeated layer by layer and the level of the liquid in container is raised after solidification of each layer. If required then some binder resin is also added to the prototype.

\section{B. SLS - Selective Laser Sintering}

SLS uses powdered materials as cartridge. This powder is spread on the platform layer by layer. Simultaneously the High Wattage laser head melts the powder to a temperature just above its melting point at the points in space to fuse the particles together and form the solid 3D object.

\section{FDM-Fused Deposition Modelling}

FDM is more convenient for users as compared to the other two types of 3D printing methods. In this method, the production material is fed to the extrusion nozzle which melts the material into semi-liquid-state and drips it across the layer as per the geometry of the prototype [2] [9]. In this paper, we will peek into the conventional method of SLS technology which is being used for 3D Metal Printing currently. Then we will discuss about the FDM technique of printing 3D metal prototypes modelled, in upcoming pics.

\section{Conventional Method of 3D PRinting}

Out of SLS, SLA and FDM, the approach was made using SLS for printing the prototypes using powdered bed. The laser acts as a power source which sinters the metal powder layer. Powdered bed is preheated to some temperature before prototyping to save energy required to fuse the particles. The 
penetration of fusing is controlled by the power supplied to the laser. It was developed and patented by Dr. Carl Deckard and academic adviser, Dr. Joe Beaman at the university of Texas in 1980s [12]. It is very costly and cannot be used as a desktop printers as per current situation, since it is not at all affordable by small scale fabricators. A thin layer of powder is applied on the platform. The laser beam then fuses the powder at the required points [3].

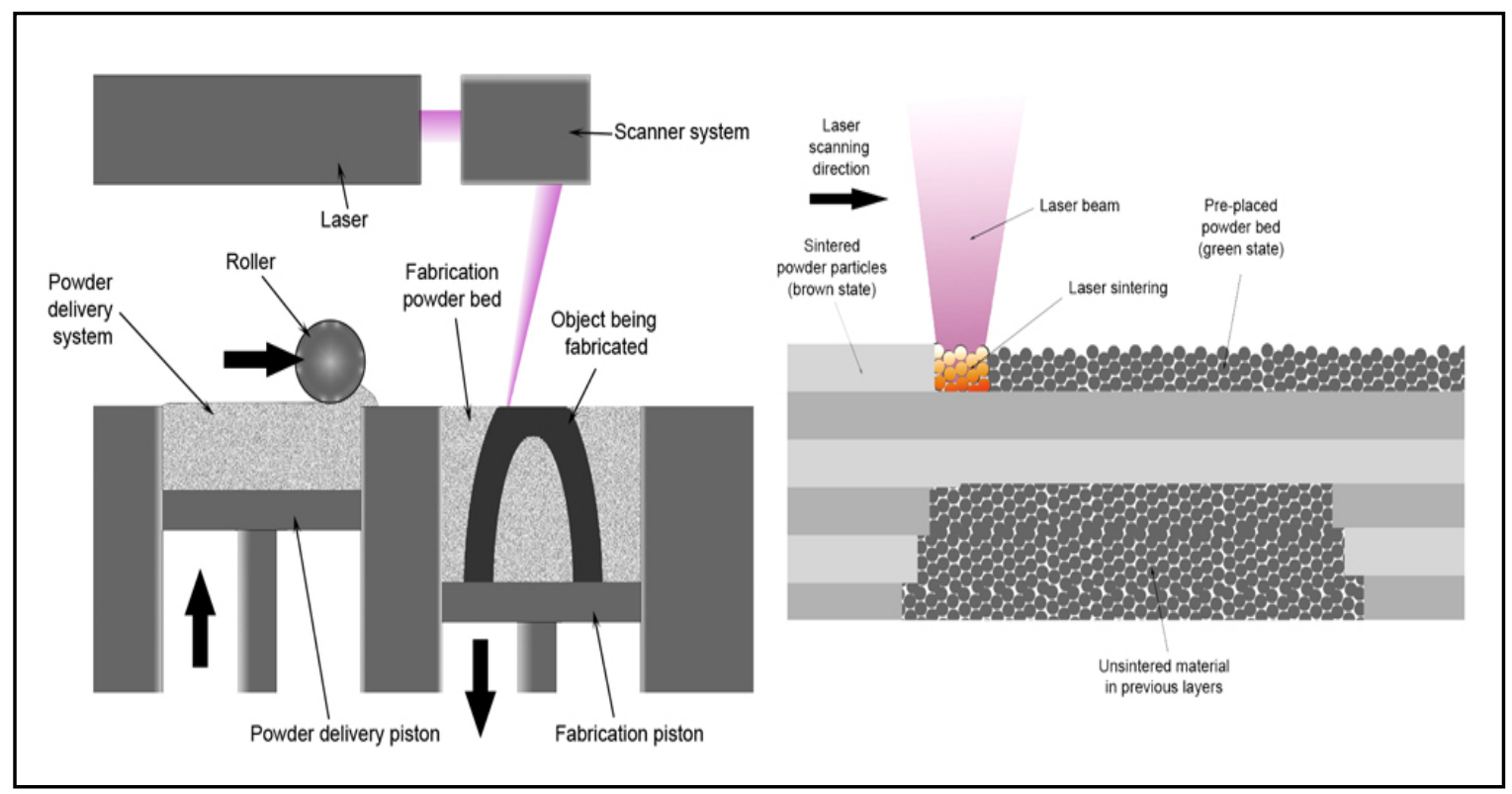

Fig. 1: Selective Laser Sintering Process (Courtesy: Materialgeeza, SLS, Wikipedia ) [12]

\section{PRINTING PROCEDURE FOR FDM 3D PRINTER}

The procedure to print the prototype using FDM printer is very simple and the following points explain how the printer shall be operated.

\section{A. Rough Grinding of Metal}

The feed for the printer can be of pure metal strips or wires which do not need to go through any grinding process or it could even be the scrap metal with homogenous internal composition. If the feed is scrap metal, then the surface exposed to impurity must be roughly grinded in order to avoid defects in rapid prototyping. When the percentage of impurity on the surface approximates to zero, then the metal is suitable for additive manufacturing of its complex shape.

\section{B. Crushing into Smaller Pieces}

The metal as a whole must not be fed to cartridge container, as the metal pieces need to be small enough to enter the funnel of nozzle and melt down for printing. Therefore it must be broken down to approximately $25 \%$ of the size of the largest diameter of nozzle funnel. Before feeding in the cartridge container, the crushed particle must be sieved through fine pores of $1 \mathrm{~mm}$ diameter and the finer particles are removed. This is done to avoid the possibility of a metal piece passing through the nozzle head without melting completely.

\section{Feed to The Induction Furnace}

The Induction furnace is the coil around the nozzle which melts the metal by creating high frequency eddy current in the metal pieces. The feed to the Induction furnace is given depending upon the level of metal in the funnel shaped nozzle of the furnace. Noticing a decrease in level, more metal pieces are fed to the Induction Furnace by pushing it through a piston in the cartridge container.

\section{Melting of Metal Pieces}

The process of melting of metals starts from the point where it is fed to the funnel of nozzle. Melting of metal pieces is controlled electronically by varying voltage and current regulators. The pieces of metal melt and flow downward.

\section{E. Chamber Vacuumisation}

The chamber is maintained at pressure lower than that of the cartridge container. This effect increases the speed of flow at which the fluid comes out. This can be controlled manually as well as automatically by the program settings that will integrate the requirement of pressure based on the density and the viscosity of the metal at the nozzle tip temperature.

\section{F. Fused Deposition Modelling of Prototype}

Now, the basic requirements of the input are filled. Then the system is controlled using the software. Based on software algorithm, head of nozzle is moved in the three axes according to the need to print the prototype required. The metal stream is protruded out of the nozzle tip on the points and lines of interest. The work can be seen developing layer by layer.

\section{G. Cooling of The Prototype}

The major objective of cooling is to enhance the quality of the product and improve the efficiency of the printer. The cooling system can be classified into two types based on their purpose. Firstly, it is used to absorb the excess heat prevailing on the outside of nozzle which increases the resistance in wires and can even damage the system. Secondly, it cools down the prototype instantly and hence allowing quick addition of the next layer of the prototype. 


\section{H. Cleansing of Nozzle}

After finishing the rapid prototyping, it is also important to clean up the nozzle. This is necessary in order to maintain the actual hole diameter of the nozzle. This is done by flushing heated compressed air at very high velocity through the nozzle.
This is required for longevity of the machine. Again the air can be heated by continuously recirculating it through the nozzle (hot Tungsten tip will heat the air repeatedly).The following diagram gives a gist of the FDM Printing Technique.

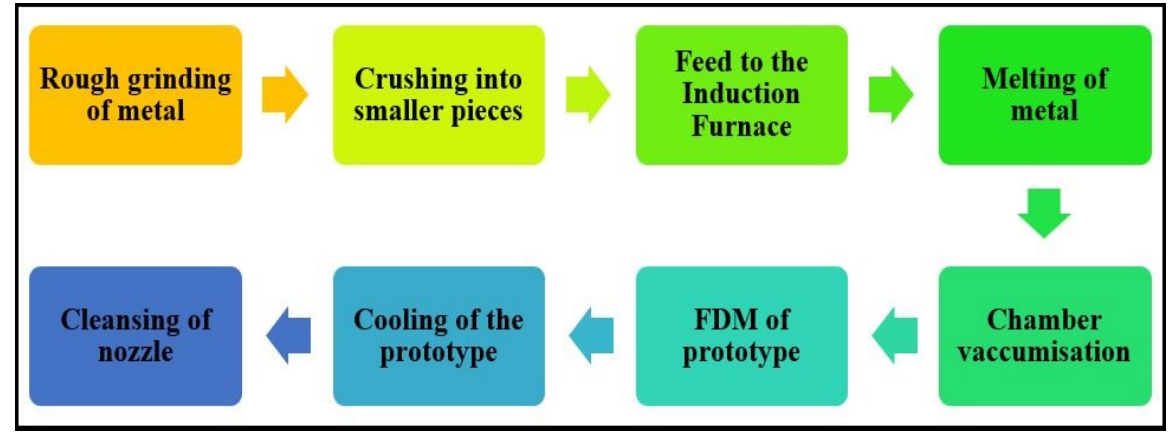

Fig. 2: Flow Chart of the Procedure for Printing Using FDM Metal 3D Printer

\section{FEED FOR THE PRINTER}

This Project has the main aim of recycling the scrap metals and forming a new prototype with the help of remolding technique. This will reduce the cost of cartridge to almost 0 . To feed the machine, initially the metals must be roughly grinded in order to remove the dust and layer of corrosion. As per the demand of the purity of the prototype, we can even purchase the pure metal strips from a hardware shop. If the desired output is an alloy, then the amount of the constituent metal can be weighed in an equal proportion and melted together to get the alloy. During this, upper nozzle will be rotated to mix the molten metal evenly.

\section{CARTRIDGE CONTAINER}

The cartridge container is a big box which will store the grinded metal pieces before the prototyping. A piston fitted within the container will be responsible for feeding these pieces into the nozzle at a constant rate and it is controlled by the software program. The inlet of the cartridge container is $25 \%$ of the size of the largest diameter of nozzle funnel. This proportion is kept to reduce the chances of big metal pieces entering into the container.

\section{INDUCTION FURNACE}

In this method, we will use an induction furnace in order to melt the metal which is more affordable than the SLS in conventional 3D printing. The Induction furnace is nothing but the coils of wire wound around the Print Head Nozzle and connected with the circuit board containing voltage and current regulator. This method is cleaner and doesn't have to come in contact with the melting body. It uses AC (Alternating Current) for melting the metal. It can be made as compact as possible of varying dimensions for various purposes suitable for industries and workshops [4]. In this, the coils will be wound around the ceramic nozzle with Tungsten tip. The heating effect produced by the induction furnace depends on the power supplied to the coil $(\mathrm{V} \times \mathrm{I})$ using alternating current. Lesser the diameter of coil, higher the amount of heat will be produced. More the number of coils packed together will also increase the thermal density.
The amount of power supplied is adjusted to melt the metal just above its melting point. Even 40 volts and 8 amps current is sufficient enough to melt aluminum at $800^{\circ} \mathrm{C}$ [7] [8]. It is simpler to control the melting process because of the electrical input. Along with this it also has higher energy efficiency.

The induction coils radiate heat perpendicular to the coil axis. This could damage the system and decrease the thermal efficiency. Therefore, the electrical wires surrounding the nozzle are kept cool by using water jacket around them [6]. The water jacket is further wrapped by insulating pads or husks to reduce thermal emissions outside the nozzle. Further the heat absorbed by this water jacket can be used to conserve energy by transforming it into other useful forms of energy.

\section{DESIGN OF PRINT HeAd NozZle}

The print head nozzle will be connected to three servo motors for the movement in all the three directions for printing. Servo motor is necessary for better precision. In order to design the nozzle it should have the following specifications:

\section{A. Nozzle's Material Property}

The nozzle is made up of ceramic tube and its tip with Tungsten which can withstand temperature up to $2000^{\circ} \mathrm{C}$. It is wrapped by induction coils. The properties of the nozzle are chosen so as to have very less coefficient of expansion. The non-reacting property of the ceramic material makes it a better choice for the application of nozzle in this model. The gap between the ceramic nozzle and coil is filled by heavy insulations.

\section{B. Nozzle Dimensions}

The nozzle is comprised of three parts as shown in Fig. 3. Ceramic nozzle with tungsten tip is fully sectioned in the diagram and represented in black. The induction coils are represented by blue colored lines around the nozzle. The tube is made of ceramic and is divided into two sections: Upper Funnel and middle Tube of hollow structure. Thickness of the ceramic tube is maintained $10 \mathrm{~mm}$ throughout its length. The first two parts of nozzle tube (Starting from the top) are of $100 \mathrm{~mm}$ each. Diameter of the topmost part of ceramic nozzle tube is $155 \mathrm{~mm}$ and gradually decreases to $40 \mathrm{~mm}$ for $100 \mathrm{~mm}$ 
length of nozzle. From here, the diameter of nozzle tube remains constant $(40 \mathrm{~mm})$ for another $100 \mathrm{~mm}$ length. The Tungsten tip of the nozzle extends for another $11 \mathrm{~mm}$. Thickness of the nozzle slightly decreases till its tip. Zooming in up to $500 \%$ of the Fig. 3 will make the dimensions of the tip clearly visible. For better understanding, another isometric view of the nozzle has been produced at the bottom right of the image in Fig. 3.

\section{Upper Funnel of Ceramic Nozzle}

The Upper Funnel is the low temperature nozzle with funnel shape, which will heat up to a temperature just above the melting point of the metal inserted. This part can be rotated with the help of motor in order to mix different metal together homogenously. It also contains spikes within the inner surface of body which enhances the property of mixing. The spikes will preferably be made up of tungsten. The diameter of the coils around this nozzle decreases as it goes down because of the funnel shape and the heating effect increases simultaneously.

\section{Cylindrical Tube of Ceramic Nozzle}

The lower part of the tube is cylindrical in shape with the main purpose of heating the metal to bring necessary fluidity to it. Therefore the length of this section is short but the coils around this nozzle are of very small diameter and even more closely packed as compared to Upper Funnel. This results in comparatively higher temperature in the tube.

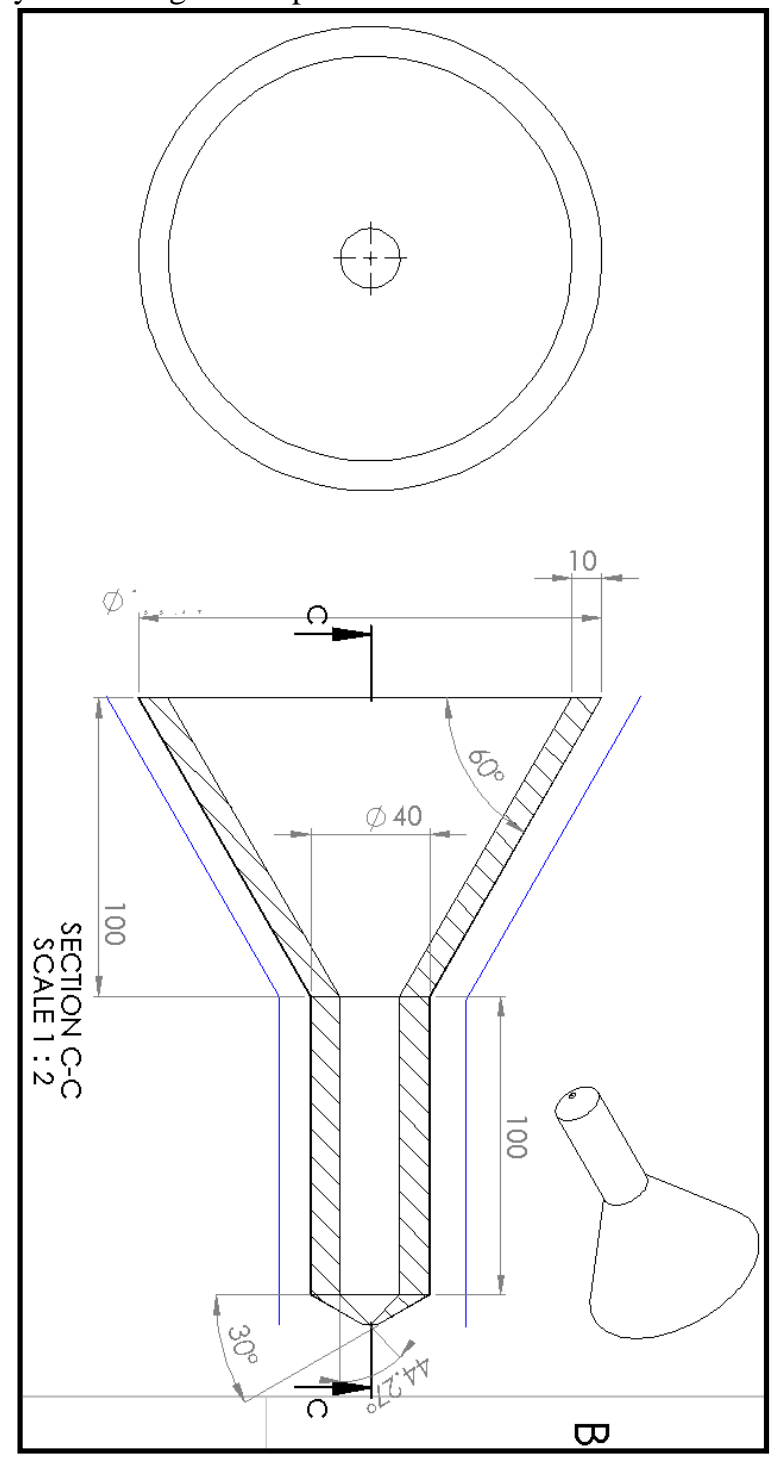

Fig. 3: Top and Sectional View of the Print Head Nozzle in FDM Metal 3D Printer

\section{E. Tungsten Tip of Nozzle}

The final nozzle is like the tip of a pen casing and is made up of tungsten. It is made to withstand high temperatures up to $3000^{\circ} \mathrm{C}$ and its machinability allows us to make fine hole at the tip of the nozzle. The diameter of the hole can be built of sizes varying from $0.25 \mathrm{~mm}$ to $0.75 \mathrm{~mm}$. Usage of Tungsten alloy just at the base instead of complete funnel and tube, saves the cost of the equipment significantly. The wires are closely wound around the center tube of ceramic nozzle and the same diameter of wires is followed till the tip. It is kept in mind that the coil does not touch the Tungsten tip of nozzle. In order to produce 
3D models of multiple material object, multiple nozzles can be equipped. For this nozzles must be integrated with the software. Three additional servo motors will be required for every nozzle, for motion in three dimensions.

\section{RAPID PROTOTYPING CHAMBER}

The chamber is closed in a hardened glass. It has slight vacuum which is used to pull the molten metal out of the chamber and speed up the process.

The chamber is filled only with the non-reactive gasses in order to avoid the possible chemical reactions of hot metal with oxygen or any other compound. The gasses will have very poor co-efficient of heat transfer.

While shifting the environment of the prototype from vacuum chamber to atmospheric condition, the bonds between the layers also increases [5]. As discussed in the topic- VII. Induction Furnace, energy absorbed by the cooling jacket can be utilized to save energy and support in creating the vacuum. For ferrous material, electromagnet can be equipped at the base to increase the rate of prototyping. This feature of enhancing the speed of fabrication can only be applied in FDM approach of 3D Metal Printing.

The platform of the chamber is maintained at low temperature and is made up of base having high thermal conductivity. Since the metals are good conductors of electricity, the heat from molten metal will immediately pass through the layers and into the base. This ensures rapid cooling of the layer imposed on the top.

After the prototype is finished, it can be easily quenched or annealed with the help of accessories involving two methods, either by dipping the prototype or spraying the fluid onto it. The quenching medium/oil can be varied according to the prototype. The specimen can be immediately dipped into the quenching medium, fixed below the printing platform. In spray type coolant, the water is sprayed onto the metal continuously to cool it down instantly.

\section{SOFTWARE MODIFICATIONS}

The software for this method of 3D printing apart from converting programmed CAD files, movement for fastest completion of the prototype and other conventional programs must be additionally programmed for few important tasks. Free open source software will be used in our project temporarily like Cura, Craftware, etc. The aims that will be focused for modification in software are as follows:

- $\quad$ Since the metal to be prototyped could be of varying melting point. Our first aim is to regulate the fluidity of the metal by changing the temperature inside the nozzle. Fluidity at varying temperatures is different for every metal. It is very difficult to determine the fluidity of the metal in hand. Therefore instead of pre-storing the fluidity data in system, fluidity of the molten metal can be identified by pouring some of the metal prior to prototyping and calibrating it according to the need.

- Unlike other printers we have to regulate the power supply to the Induction furnace. Therefore, the second requirement is to control the power input to the induction furnace.

- $\quad$ The third most important aim of this prototype is to create a balance between the vacuum pressure, gravity force and magnetic force acting on the fluid to regulate its flow.

\section{COST ANALYSIS}

The following Fig. 4 explains about the cost invested in the 3D Metal Printing. The purpose of using 3D metal printing could vary in various aspects of industry or society. It can be used for printing the pattern for the casting process of complex shapes. It also has application in high-end automobiles, consumer goods and aerospace.

When we think of commercialization of 3D Metal Printing, the cost is the major drawback which stops it from acting as a Desktop Printer. People with small workshop won't be able to afford these printers. In order to suit the need of fabricators in India, FDM stands as the best option for 3D metal Printing.

\begin{tabular}{|c|c|}
\hline 3D Printing Machine & $\$ 750,000$ \\
\hline Vacuum Furnace & $\$ 500,000$ \\
\hline Total Capital & $\$ 1,300,000$ \\
\hline Capital Declaration - 7 Years & $\$ 187,714$ \\
\hline Gas and Electric per Year & $\$ 75,000$ \\
\hline Maintenance per Year & $\$ 102,500$ \\
\hline Labor and Overhead per Year & $\$ 180,000$ \\
\hline Total Non-Material per Year & $\$ 543,214$ \\
\hline Total Pounds per Year at 20hrs/5days/50weeks & $\$ 47,414$ \\
\hline Non-Material Cost per Year & $\$ 11.46$ \\
\hline Material Cost per Pound Produced & $\$ 18.69$ \\
\hline Total Production Cost per Pound & $\$ 30.14$ \\
\hline \multicolumn{2}{|c|}{$\begin{array}{l}\text { Shop Operating Cost Model } \\
\text { Assumes R10 machine with } 40 \times 20 \times 10 \text {-inch build box and metal } \\
\text { printed in } 33 \% \text { of the build area }\end{array}$} \\
\hline
\end{tabular}

“Cost model for ProMetal's R10 3D printer includes machine and furnace capital depreciation, labor, overhead, utilities, maintenance, and materials. (Source: Assembly Automation, Volume 23 - Number 4 - 2003 - pp. 340-345.)” [9].The cost comparison between FDM and SLS technology of 3D Metal Printing is made below:

- $\quad$ Fitting a CO2 or Nd-YAG laser costs around $\$ 800$ which is 10 times as much as an induction furnace [10]. Thus making the initial investment for the printer not affordable.

- Even the Research \& Development is responsible for the higher cost of SLS technology wherein the calibration of the laser with the metal powdered cartridge plays a very important role. On the other hand, there is very less requirement of R\&D in FDM technology of 3D Metal Printing as compared to SLS.

- The cost directly doubles when advance prototyping such as multiple metal fabrications is required. This is so, because spreading of different metal powder on the bed and altering their power of fusion to which the metal layers are fused together using the laser is a very complex and difficult process. 
- The metal powder cartridge used here is manufactured only in few countries worldwide, with a very high cost. Again the cost of the metallic powder varies with the type of metal required. The companies have monopoly, since the printer will accept the cartridge made from its own company. The FDM technology enables the use of pure metals and even the scrap metals also, depending on our requirement.

\section{AdVANTAgES AND Disadvantages OF FDM Over SLS 3D METAL PRINTING}

\section{A. Advantages of FDM over SLS Technology for 3D Metal Printing.}

- Looking down through the conventional method of 3D Metal Printing using SLS technology is much costlier than FDM technology.

- Even fabrication becomes more difficult when the combination of different metals comes into SLS approach.

- We can easily see that FDM utilizes the Recycle principle, where the same raw material can be used multiple times. This is one of the major advantages of FDM over SLS for 3D Metal Printing. Imagine a case where you have the capability of forming a prototype fulfilling some purpose and then immediately that same raw material can be rebuilt for some other purpose. For instance, you produced a gold ornament of intricate shape. Now if the user wants to have it remodeled in some other shape, then he will not be able to do so as per the current technology of 3D metal printing. That person will have to buy a new set of gold cartridge for preparing the new shape, whereas this drawback is covered in FDM technique of 3D metal printing. It reduces the cost of the raw materials.

- Even the net weight of the project becomes very less as compared to conventional technique.

- A person working in remote place (with minimal need of power supply) could also make use of this printer efficiently with the help of just the scrap metal.

- It is impossible to create a design which is hollow from inside and completely enclosed from outside using the conventional method, since the metal powder inside the chamber cannot be removed after completion. This drawback is not seen in FDM Technique.

- The strength of the prototype can be varied by using different heat treatment processes immediately after the prototyping finishes.

- Another great use of this technology is in space where strong metallic tools are needed and the weight of equipment must be limited. The tools can be formed whenever required and again it can be reshaped for some other purpose without carrying additional cartridge for the job.

B. Disadvantages of FDM over SLS Technology for 3D Metal Printing

- The heat is slightly radiated in the environment due to the Induction Coils. Although the amount of heat dissipated through the coils will be very less and can be easily controlled by the cooling jacket.

- Another disadvantage is that FDM technique requires support materials for modelling of prototypes having outward ascend angle beyond $45^{\circ}$. For this even the support material should be strong enough to withstand the heat. It should also be easily detachable. This drawback can be resolved by again using ceramic support with facing sand layer on top of it.

- It is also quite slower than conventional technique. It takes about 10-15 minutes to heat up the nozzle/induction furnace before starting the project. New advancements and further research can be done on this to increase the speed of FDM technology.

Apart from these basics, further research will be conducted on making this technology sufficient enough to work on batteries so as to enable its use even in the remote areas where there's no power supply for operating machines like this. Currently there is no technology which can easily purify molten metals within the Induction Furnace. In the future inventions, when it becomes possible for us to do so, then that technology can be applied in FDM model also.

\section{CONCLUSION}

The theoretical concept behind FDM additive manufacturing method for 3D Metal Printing was prepared. The scope for this method is very large and affordability is the key feature of this 3D Metal Printer. The conventional method was briefly compared with FDM design. All the components of this printer were explained briefly emphasizing the need of the Printer and methods to overcome them. The cost analysis among the conventional SLS method with the proposed FDM method was summarized. Finally, a gist of the possible applications and future scope of this project was discussed.

\section{ACKNOWLEDGMENT}

I have to be very much thankful to the almighty who has showered me with the hope and confidence to step ahead in this part of research which has not yet been covered because of its difficulties. I am very grateful to my project guide Sriram Chauhan Sir for helping me all the way to complete my research paper. His suggestions has lightened up my way even in the darkest time. With my whole heart, I will thank my parents for their unconditional support towards me in every step that I took ahead. I am very fortunate to have friends who are capable in all the various fields and cherish me with them despite of my eccentricities. It was because of these people who assisted me by their comments, remarks, compliments, and unavoidable questions to make my research paper better every time.

\section{REFERENCES}

[1] Nale, B. Swati and A.G. Kalbande, "A Review on 3D Printing Technology".

[2] David Bak, "Rapid prototyping or rapid production? 3D printing processes move industry towards the latter”, Assembly Automation, Vol. 23, No. 4, Pp.340-345, 2003.

[3] L. Dong, N. Barth, J.P.M. Correia and S. Ahzi, "Modeling and numerical simulation of selective laser sintering", 2016 17th International Conference on Thermal, Mechanical and Multi-Physics 
Simulation and Experiments in Microelectronics and Microsystems (EuroSimE), Montpellier, Pp.1-4, 2016.

[4] Kulkarni, Uma, S. Jadhav and M. Magadum, "Design and control of medium frequency induction furnace for silicon melting”, International Journal of Engineering Science and Innovative Technology (IJESIT), Vol. 3, No. 4, 2014.

[5] X.P. Niu, B.H. Hu, I. Pinwill and H. Li, "Vacuum assisted high pressure die casting of aluminium alloys", Journal of Materials Processing Technology, Vol. 105, No. 1-2, Pp. 119-127,2000.

[6] S.L. Semiatin, "Elements of induction heating: design, control, and applications”, ASM International, 1988.

[7] http://www.instructables.com/id/7-Uses-For-an-Induction-HeatingMachine-How-to-Mak/

[8] http://www.rmcybernetics.com/projects/DIY_Devices/diy-inductionheater.htm

[9] https://www.3dsupplyguys.com/3d-printing-education-center/fdm-vssla-vs-sls/

[10] https://www.realself.com/Yag-laser/reviews

[11] http://www.lboro.ac.uk/research/amrg/about/the7categoriesofadditivema nufacturing/

[12] https://en.wikipedia.org/wiki/Selective_laser_sintering 